\title{
Multi-Modality Imaging of Drug-Eluting Stent-Related Multi-Vessel Coronary Aneurysms Presenting as Recurrent Stent Thrombosis
}

Gunasekaran Sengottuvelu, MD; Ravindran Rajendran, MD; Immaneni Sathyamurthy, MD

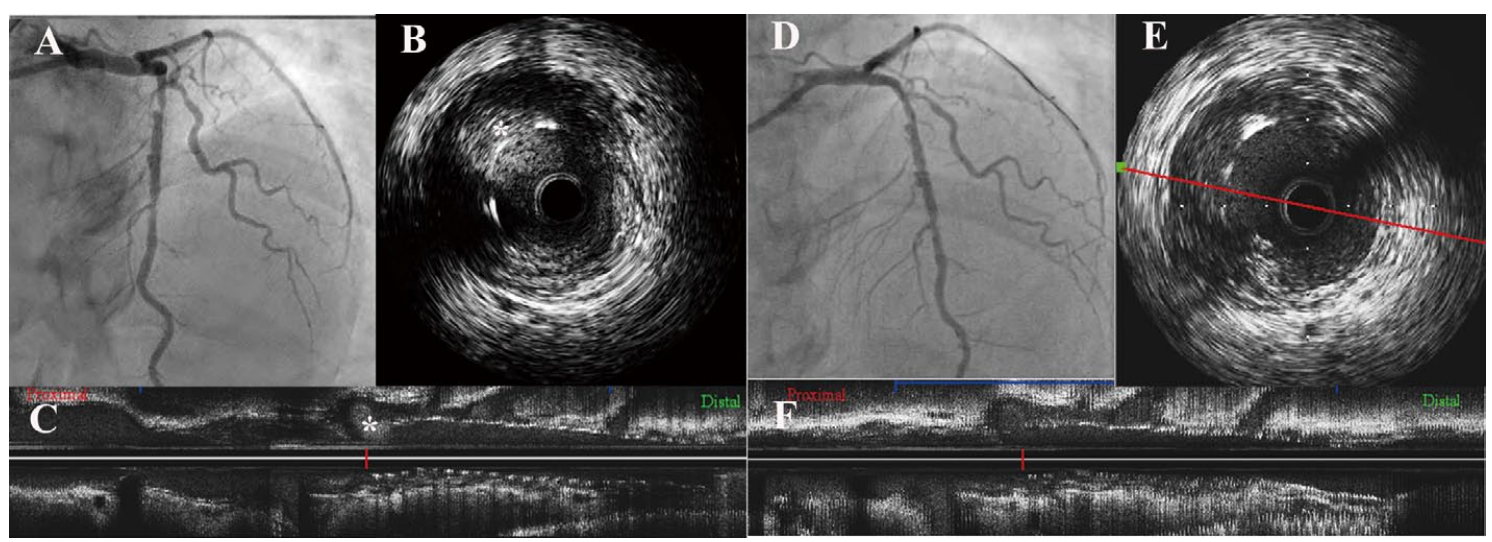

Figure 1. (A) Angiogram showing multiple aneurysms in the stented mid-left anterior descending coronary artery (mid-LAD) and a critical narrowing at the proximal edge of the stent. (B,C) Longitudinal and axial intravascular ultrasound (IVUS) showing late acquired malapposition with thrombus $\left({ }^{*}\right)$ protruding into the lumen from the aneurysmal cavity. (D) LAD angiogram after ticagrelor and enoxaparin showing improvement in the severity of the narrowing. (E,F) Corresponding longitudinal and axial IVUS showing partial resolution of the thrombus.

A 52-year-old hypertensive woman had undergone multi-vessel percutaneous coronary intervention (PCI) with 3 everolimus-eluting Xience stents (EES) to the left anterior descending artery (LAD), left circumflex coronary artery (LCX) and right coronary artery (RCA) and was on aspirin plus ticagrelor. She did well at 1-year follow-up with normal echocardiography and stress-test. Ticagrelor was discontinued at 1 year and dual anti-platelets with aspirin and clopidogrel were started in view of the multiple stents. Ten days after switching, she presented with effort angina, normal rest electrocardiogram and negative cardiac bio-markers. Coronary angiogram indicated multiple aneurysmal dilatations within the LAD and LCX stents (which were smooth at the end of index PCI) along with a $99 \%$ stenosis just proximal to the LAD stent with TIMI-3 flow (Figure 1A; Movie S1). Intravascular ultrasound (IVUS) showed multiple aneurysms over the LAD stent with huge thrombus (Figure 1B,C; Movie S1) protruding from the aneurysm into the lumen proximal to the stent (which appeared as a tight lesion on angiogram). Thrombus aspiration did not help and the patient was treated with enoxaparin, aspirin and ticagrelor. She had remarkable improvement in effort tolerance, and angiogram after 4 weeks showed significant resolution of the narrowing (Figure 1D; Movie S1). IVUS of the LAD (Movie S1; Figure 1E,F) showed multiple aneurysms with acquired malapposition and significant resolution of the thrombus. Angiogram and IVUS of the LCX also showed aneurysms. The patient was maintained on aspirin and ticagrelor even though cytochrome P450 2C19 genotyping for clopidogrel resistance was negative $\left(* 1 /{ }^{*} 1\right)$, and was kept on close follow-up. Six months later the patient again presented with class III effort angina while on DAPT and had new-onset regional wall motion abnormality. Angiogram showed

Received July 12, 2016; revised manuscript received September 13, 2016; accepted September 30, 2016; released online October 29, 2016 Time for primary review: 26 days

Department of Cardiology, Apollo Hospitals, Chennai, Tamil Nadu (G.S., R.R., I.S.); Cardiology Department, Chennai Medical College (under Dr. MGR University), Tiruchirappalli, Tamil Nadu (R.R.), India

Mailing address: Gunasekaran Sengottuvelu, MD, DM, FRCP, Department of Cardiology, Apollo Hospitals, Chennai 06, Tamil Nadu, India. E-mail: drgseng@gmail.com

ISSN-1346-9843 All rights are reserved to the Japanese Circulation Society. For permissions, please e-mail: cj@j-circ.or.jp 


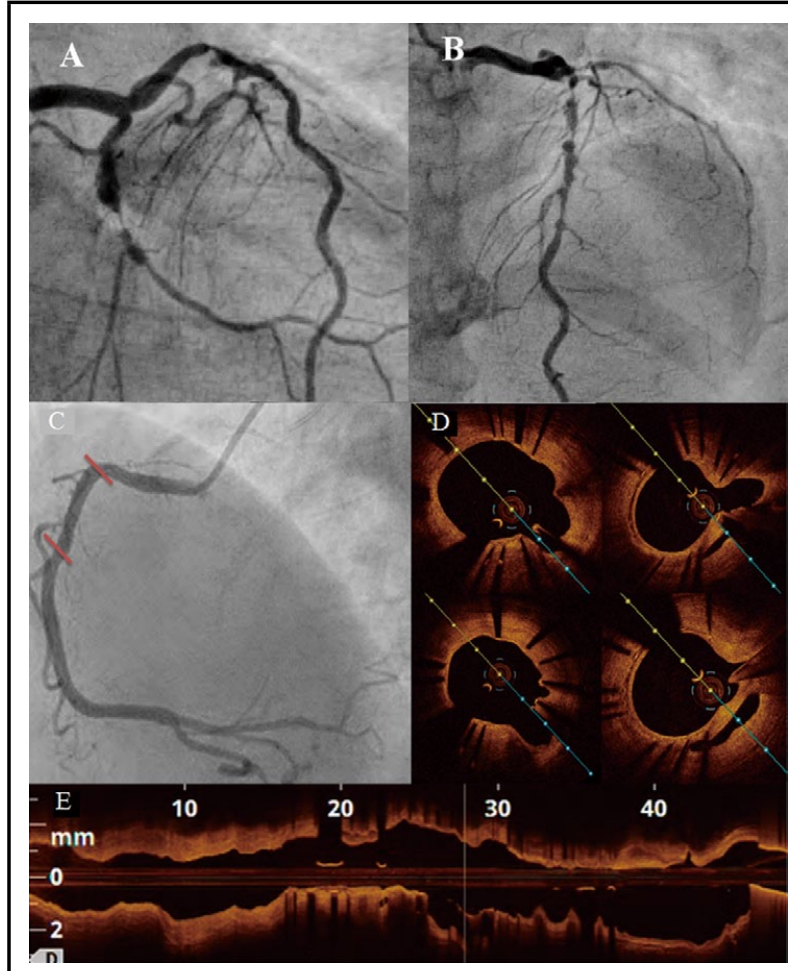

Figure 2. (A,B) Left coronary artery in (A) cranial and (B) caudal view showing critical narrowing across the left anterior descending and left circumflex coronary artery stents. (C) Right coronary artery with a patent stent in the proximal segment. (D,E) Axial and longitudinal optical coherence tomography showing aneurysms and thrombus.

critical narrowing of both the LAD and LCX stents with aneurysms and thrombus (Figure 2A,B; Movie S1). Optical coherence tomography (OCT) of the angiographically normal RCA was striking, with multiple aneurysms and thrombus within the aneurysmal dilatation (Figure $\mathbf{2 C}-\mathbf{E}$; Movie S2). The patient underwent coronary artery bypass surgery with grafts to the LAD and major OM.

Multiple aneurysms after EES are rare and are presumably due to polymer hypersensitivity. The management of this condition is challenging. ${ }^{1}$ IVUS showed thrombus, which favored medical therapy with anti-coagulants and anti-platelets over repeat stenting in the first episode. At the second episode of stent thrombosis, coronary artery bypass surgery was a good option because both stents had subocclusive thrombus. OCT visualized the aneurysms filled with thrombus in the RCA that were not seen on angiography. ${ }^{2,3}$ Coronary major evagination with multiple interstrut hallows was the possible differential diagnosis for this OCT finding, but this is usually procedure related and noted immediately after PCI as peri-stent staining, and is unknown with the present-generation EES.,5

\section{Disclosures}

None to declare.

\section{References}

1. Aoki J, Kirtane A, Leon MB, Dangas G. Coronary artery aneurysms after drug-eluting stent implantation. JACC Cardiovasc Interv 2008; 1: 14-21.

2. Kang SJ, Lee CW, Song H, Ahn JM, Kim WJ, Lee JY, et al. OCT analysis in patients with very late stent thrombosis. JACC Cardiovasc Imaging 2013; 6: 695-703.

3. Sengottuvelu G, Rajendran R. Multimodality imaging in the evaluation of recurrent very late stent thrombosis. Asia Interv 2015; 1: 124

4. Tada T, Kadota K, Hosogi S, Kubo S, Ozaki M, Yoshino M, et al. Optical coherence tomography findings in lesions after sirolimus-eluting stent implantation with peri-stent contrast staining. Circ Cardiovasc Interv 2012; 5: 649-656.

5. Radu MD, Räber L, Kalesan B, Muramatsu T, Kelbæk H, Heo $\mathrm{J}$, et al. Coronary evaginations are associated with positive vessel remodeling and are nearly absent following implantation of newer-generation drug-eluting stents: An optical coherence tomography and intravascular ultrasound study. Eur Heart $J$ 2014; 35: 795-807.

\section{Supplementary Files}

Supplementary File 1

Movie S1. Left coronary artery (LCA) angiograms and intravascular ultrasound (IVUS) after index percutaneous coronary intervention (PCI), during ST, and at 1-month and 6-month follow-up (caudal and cranial). ACS, acute coronary syndrome; LAD, left anterior descending coronary artery; LCX, left circumflex coronary artery; LMWH, low-molecular-weight heparin.

Supplementary File 2

Movie S2. Follow-up angiogram of the right coronary artery.

Please find supplementary file(s);

http://dx.doi.org/10.1253/circj.CJ-16-0654 